\title{
Load Compensation in Mechatronic System with Observer
}

\author{
Aleksey Kolganov (Professor, Ivanovo State Power University - ISPU), \\ Sergey Lebedev (Associate professor, ISPU), Mikhail Kulenko (Associate professor, ISPU)
}

\begin{abstract}
Features of combined mechatronic systems creation with a rigid mechanics are observed in details. The usage of astatic regulators for the basic positioning loop is offered. Efficiency of regulators and observers adjustment on Bessel polynomial is shown for mechatronic positioning systems. Outcomes of synthesis of the load observer taking into account the drive dynamics and different forms of load in the system are resulted.
\end{abstract}

Keywords - Motor drives, observers, mechatronics, motion control, computer simulation.

\section{INTRODUCTION}

Mechatronic units on the basis of alternative current motors, frequency converters and microprocessor controllers allow the implementation of specified statics and dynamics; it is essential to expand a passband, thus ensuring the technology equipment in competitive performances [1], [2]. The application of mechatronic modules in the systems of positioning for machine-tool industry and robotic technology is especially actual. Thus the important factor of influence on each axis characteristics is kinematic and dynamic connections between axes as variable loads of the complex form.

Mainly the control theory deals with two basic principles of control: an error-feedback control and a disturbance compensation control. Control systems of electric drives were under construction on the basis of usage of error-feedback control principle at creation of torque, speed and position regulating circuits, and influence of load on performances was defined by rigidity of speed-torque characteristics at usage of static regulators.

In this case astatic regulators ensure control of a static error only. Alternative principle (the disturbance-compensating control) was not applied widely because of complexity of compensation circuit engineering implementation and disturbances determination.

Implementation of compensating connection on disturbances on the basis of usage of load observers [2] allows to start the practical usage of combined control ensuring the control of dynamic processes on disturbance.

\section{STRUCTURE OF MECHATRONIC SYSTEM WITH LOAD COMPENSATION}

The mechatronic systems structure with combined control (fig. 1) involving both control principles within maximum range is offered. The astatic position regulator (APR) for maintenance of regulation quality on control signal and absence of a static error on disturbance signal is used. Also the load observer ( $L O)$ is applied for making a compensation unit ( $C U$ ) to compensate disturbances of the arbitrary form.

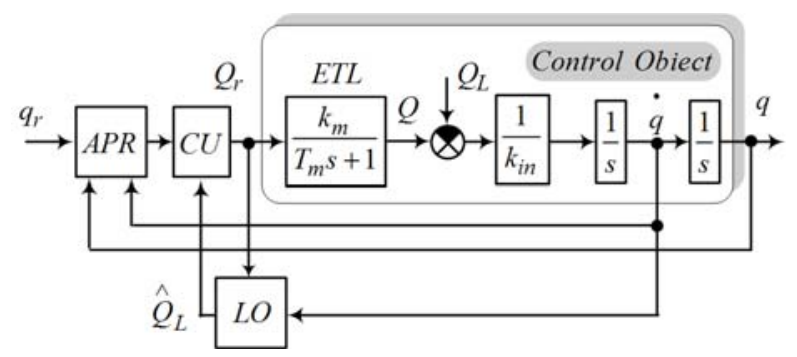

Fig. 1. Structure of mechatronic system with combined control and load compensation.

The control object taken as a principle structures represents a mechanical part of mechatronic unit of linear or angular motion $(q)$ combined with the "rigid" mechanics characterized with the inertia coefficient $\left(k_{i n}\right)$ and disturbance - mechanical load $\left(Q_{L}\right)$.

The mechanics is actuated by the electromagnetic torque of the drive $(Q)$, formed in mechatronic unit of vector control system by reference signal $\left(Q_{r}\right)$. The electromagnetic torque loop (ETL) corresponds to aperiodic block with parameters $\left(k_{m}, T_{m}\right)$. The torque reference signal is formed by a regulator and compensation unit under the signal for position $\left(q_{r}\right)$, position feedback $(q)$, speed $(q)$ and an estimation $\left(\hat{Q}_{L}\right)$ of the load observer $(L O)$.

\section{AdJUSTMENT OF SYSTEM DYNAMICS}

The system dynamics, both position regulating loop and load estimation, is defined by the passband matching to the demands of technology and form of dynamic characteristic.

The dynamic characteristic aspect is defined [3] with the chosen distribution of roots of the characteristic equations for the closed system of regulating and the load observer.

Owing to a monotonicity of processes in positioning systems traditionally the allocation of roots on a binominal formula, and in observers to high-speed performance distribution of roots on Butterworth polynomial are applied [3] $-[5]$.

Also it is necessary to observe Bessel polynomial $[5,6]$ characterized by almost constant group delay across the entire passband

$$
D(\omega)=-\frac{d}{d \omega} \varphi(\omega) \approx 1
$$


According to expression (1) all harmonics in a passband transit through dynamic system with an equal delay thus preserving the wave shape of filtered signals in the passband. Bessel filters are often used in audio crossover systems.

The use of Bessel polynomial for mechatronic positioning units and load observers is proposed in this paper. For the systems with order $n=3$ characteristic polynomials of distributions look like:

- binomial distribution (Newton binom)

$$
s^{3}+3 \omega_{0} s^{2}+3 \omega_{0}^{2} s+\omega_{0}^{3}
$$

- Butterworth polynomial distribution

$$
s^{3}+2 \omega_{0} s^{2}+2 \omega_{0}^{2} s+\omega_{0}^{3}
$$

- Bessel polynomial distribution

$$
s^{3}+3.41 \omega_{0} s^{2}+4.87 \omega_{0}^{2} s+2.77 \omega_{0}^{3},
$$

where $\omega_{0}$ is a center root.

Polynomials (2), (3) and (4) are normalized on the frequency $\omega_{0}=\omega_{-3 \mathrm{~dB}}$ ensuring peak attenuation in a passband $-3 \mathrm{~dB}$. The fragment of time responses for different distributions at unit step signal $(t=1 \mathrm{~s})$ is shown in fig. 2 .

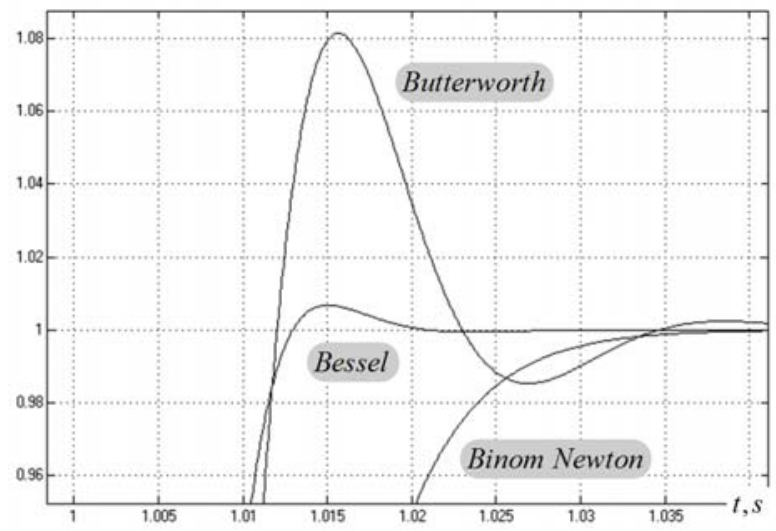

Fig. 2. Time responses of roots distributions.

As shown in [6], with growth of system order the Butterworth distribution is characterized with overshoot and settling time increase.

In the case of Bessel polynomial use the overshoot is within $1 \%$, and as for speed of response the Bessel distribution leads "competitors" in 2-3 times.

\section{Astatic Position Regulator Synthesis}

Let's execute synthesis of a closed loop of position regulating for an astatic position regulator in the form of modified PID controller with the compensation filter of nulls of a closed-loop transfer function (fig. 3).

Such modification of PID controller is admitted for positioning systems when $q_{r}(t)=$ const . It should be noted we accept the torque loop transfer function as scaleable coefficient because the time constant $T_{m}$ is negligible in comparison with mechanical processes.

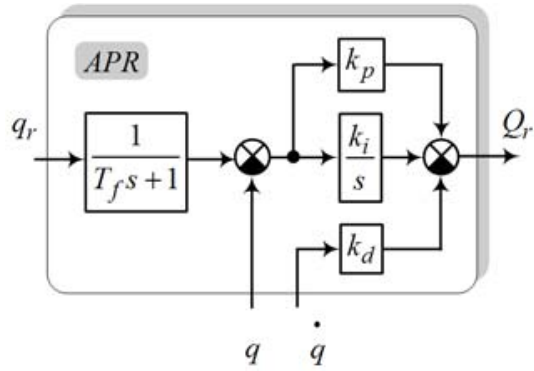

Fig. 3. Astatic position regulator structure.

To synthesize the controller parameters for the given passband $\left(f_{p r}\right)$ it should be defined $\omega_{0}=2 \pi f_{p r}$. Then the controller parameters have the form:

- binomial distribution (Newton binom)

$$
k_{p}=3 \omega_{0}^{2} k_{\text {in }} ; k_{i}=\omega_{0}^{3} k_{\text {in }} ; k_{d}=3 \omega_{0} k_{\text {in }} ; T_{f}=\frac{3}{\omega_{0}} ;
$$

- Butterworth polynomial distribution

$$
k_{p}=2 \omega_{0}^{2} k_{i n} ; k_{i}=\omega_{0}^{3} k_{i n} ; k_{d}=2 \omega_{0} k_{i n} ; T_{f}=\frac{2}{\omega_{0}} ;
$$

- Bessel polynomial distribution

$$
\begin{aligned}
k_{p}=4.87 \omega_{0}^{2} k_{\text {in }} ; k_{i} & =2.77 \omega_{0}^{3} k_{\text {in }} ; k_{d}=3.41 \omega_{0} k_{\text {in }} ; \\
T_{f} & =\frac{4.87}{2.77 \omega_{0}} .
\end{aligned}
$$

It is possible to use Simulink Matlab for simulation of three astatic systems with regulator parameters defined in expressions (5), (6), (7), and the same parameters of control object, passband, positioning and disturbance signals.

The transient processes for all systems combined in one graph are shown in fig. 4. The step of positioning signal happens at time moment $t=0$, and the growth of load takes place at $t=0.3 \mathrm{~s}$.

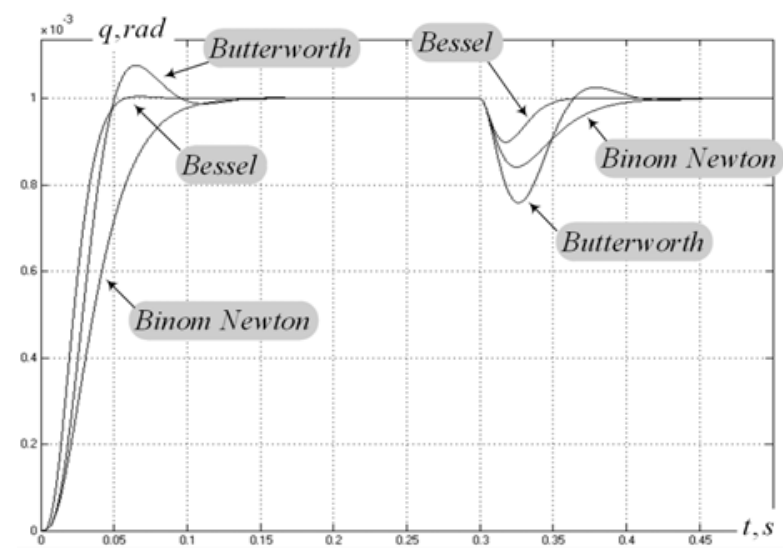

Fig. 4. Astatic system transient processes. 
Analyzing simulation results it is possible to say that the astatic system adjusted according to Bessel polynomial has the best performance. Besides, along with the best response time on reference signal, advantage in speed performance and a minimum dynamic error on disturbance is attained.

\section{LOAD OBSERVER AND COMPENSATION UNIT SYNTHESIS}

The usage of astatic state observers [7], [8] as load observers is offered. In this case it is the 1st order astatic load observer taking into account the model of drive dynamics. The speed should be chosen as a system output parameter to reduce the order of the observer. The basis model of control object improved by disturbances model for the load observer synthesis is shown in fig. 5.

Load observer state equation:

$$
\frac{d}{d t}\left[\begin{array}{c}
\hat{q} \\
\hat{Q} \\
\hat{Q}_{L}
\end{array}\right]=\mathbf{A}\left[\begin{array}{c}
\hat{\dot{q}} \\
\hat{Q} \\
\hat{Q}_{L}
\end{array}\right]+\mathbf{B} Q_{r}+\left[\begin{array}{c}
l_{1} \\
l_{2} \\
l_{3}
\end{array}\right](q-\dot{q}),
$$

where $\mathbf{A}=\left[\begin{array}{ccc}0 & \frac{1}{k_{i n}} & \frac{-1}{k_{i n}} \\ 0 & \frac{1}{T_{m}} & 0 \\ 0 & 0 & 0\end{array}\right] ; \mathbf{B}=\left[\begin{array}{c}0 \\ \frac{k_{m}}{T_{m}} \\ 0\end{array}\right]$.

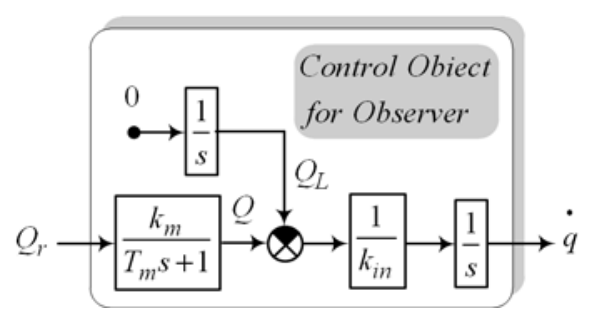

Fig. 5. Structure of control object expanded by disturbances model.

In accordance with (8) the load observer structure (fig. 6) is obtained.

To provide dynamics of the observer corresponding to Bessel distribution the observer parameters are the following:

$$
\begin{gathered}
l_{1}=3.41 \omega_{0 L}-\frac{1}{T_{m}} ; \\
l_{2}=4.87 \omega_{0 L}^{2} k_{i n}-2.77 \omega_{0 L}^{3} k_{i n} T_{m}-3.41 \omega_{0 L} \frac{k_{i n}}{T_{m}}+k_{i n} \frac{1}{T_{m}^{2}} \\
l_{3}=-2.77 \omega_{0 L}^{3} k_{i n} T_{m},
\end{gathered}
$$

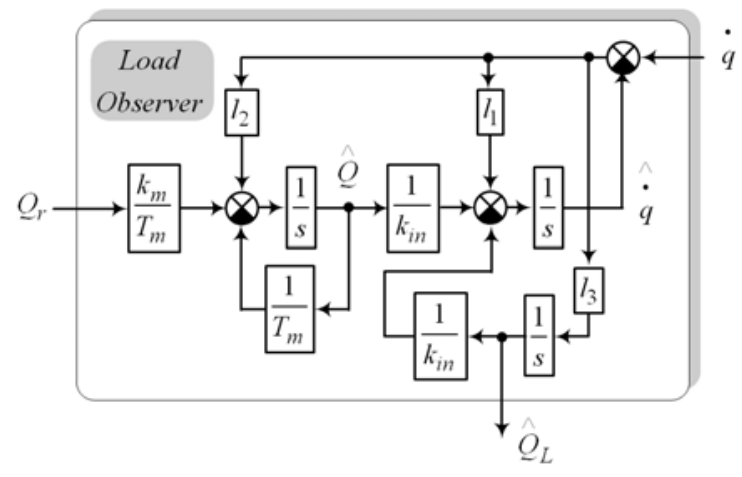

Fig. 6. Load observer structure.

where $\omega_{0 L}=5 \omega_{0}-$ a center root of load observer characteristic equation. Basic elements of the load compensation unit are the following (fig. 7): the drive dynamics compensation unit $(D D C)$ and zeros observer dynamics compensation unit (ODC).

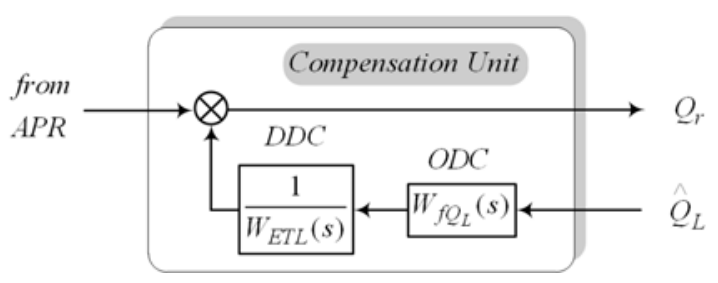

Fig. 7. Compensation unit Structure.

The transfer function of $D D C$ unit is inverse transfer function of drive electromagnetic torque loop (ETL), as a result it is gained:

$$
W_{D D C}(s)=T_{m} s+1 .
$$

Performing the joint analysis of control object and the load observer, it is possible to define a transfer function between load and its estimation:

$$
\frac{\hat{Q}_{L}(s)}{Q_{L}(s)}=\frac{-l_{3}\left(T_{m} s+1\right)}{s^{3} k_{i n} T_{m}+s^{2}\left(k_{i n}+k_{i n} l_{1} T_{m}\right)+s\left(k_{i n} l_{1}+l_{2} T_{m}-l_{3} T_{m}\right)-l_{3}} .
$$

For zeros compensation in (13) the transfer function $O D C$ must be the following:

$$
W_{f Q_{L}}(s)=\frac{1}{T_{m} s+1} \text {. }
$$

As units with transfer functions (12) and (14) are included in series connection, the load compensation loop in the unit $C U$ will be inertialess.

\section{AstatiC AND COMBINED SYSTEMS RESEARCH}

Joint simulation in Simulink Matlab of the astatic and combined systems shows (fig. 8) that the load compensation loop does not influence the positioning processes (control signal), but effectively reduces a dynamic error at a load step. 


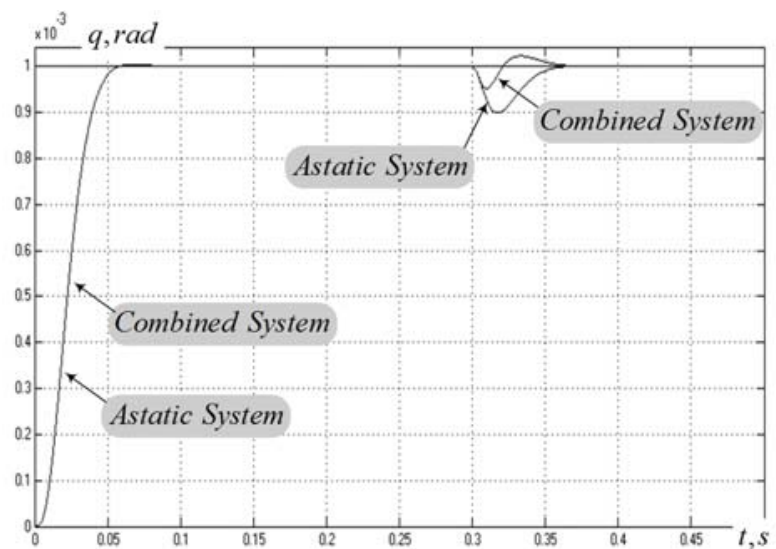

Fig. 8. Comparing of transient processes in systems.

As in real mechatronic systems the load form can be arbitrary enough, the systems response at the zero reference signal for positioning was researched. Fig. 9 shows:

1. Constant load value (both control systems give a zero steady state error);

2. Linear increasing of load (the error misses in combined system, the astatic system has a constant value of error);

3. Variation according to parabolic law (the error misses in combined system, in astatic, error is permanently growing);

4.Harmonic load variation with constant amplitude and frequency (the astatic has a higher error than the combined).

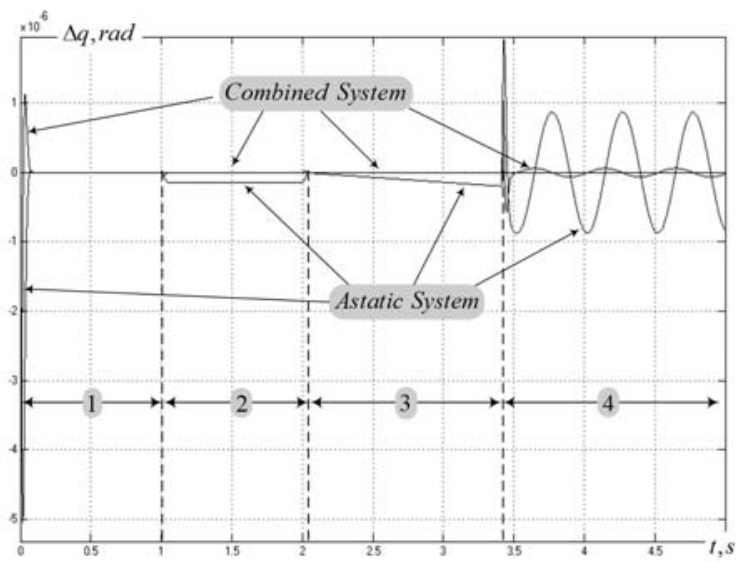

Fig. 9. Influence on an error value of different forms disturbances.

\section{CONCLUSION}

The following results were obtained:

- The efficiency of usage of astatic regulators on the basis of modified PID controller with the input filter for the combined mechatronic position systems was shown;

- The efficiency of usage for mechatronic systems dynamic adjustment of Bessel polynomial distribution both for regulators and observers was shown;

- The structure and design procedure for the 1st order astatic load observer taking into account the rigid mechanics drive dynamics was proposed;

- The comparative analysis of a "classical" astatic system properties and offered combined control structure under the condition of disturbances of the various forms is performed.
The results of simulation experiment validate theoretical conclusions. All of the proposed structures are microprocessor realizable and can be extended to mechatronic systems of different configuration and application.

\section{REFERENCES}

[1] N. Gnezdov, A. Kolganov, S. Lebedev. Application of astatic state observers in electromechanotronic modules, in Scientific Journal of Riga Technical University, vol. 27, pp. 151-154, Riga, 2010.

[2] A. R. Kolganov, S. K. Lebedev, N. E. Gnezdov, «State and load observers of modern electromechanical systems» in Proceedings of Tula State University, vol. 3, part 2 pp.18-23, Tula, 2010.

[3] N. T. Kuzovkov, Modal control and observers. Moscow: Machine building, 1976.

[4] A. R. Kolganov, S V. Burenin, Algorithms and functional design programs of control systems of electromechanical objects/Ivanovo: Ivanovo State Power University Press, 1997.

[5] Moshits George, Horn Peter. Designing of active filters. - M: World, 1984.

[6] N. Gnezdov, A. Korotkov and S. Lebedev «Selecting the parameters of standard distributions in electric drives synthesis» in ISPU Vestnic, vol. 3, pp.14-16, 2008.

[7] S. K. Lebedev, A. A. Korotkov «Algorithms of synthesis for the electric drives load observers» in ISPU Vestnic, vol. 3, pp.5-8, 2009.

[8] A. R. Kolganov, S. K. Lebedev, N. E. Gnezdov, «Microprocessor implementation of state and load observers for electromechanotronic modules» in ISPU Vestnic, vol. 2, pp.66-68, 2010.

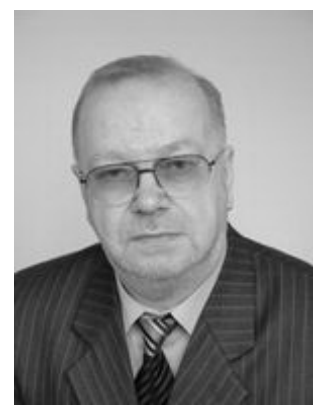

Aleksey R. Kolganov in 1974 received Engineer's degree in Ivanovo Power Institute. In 1981 he received Candidate's degree in electrical engineering for the dissertation "Computer-Aided System Design for Modeling of Electric Drives". In 2000 he received Doctor's degree for the dissertation "Technology of Automation of Functional Designing and Analysis of Complex Dynamic Objects (for Example of Electromechanical Systems)".

Head of Electric Drive and Automation of Industrial Plants Department, Ivanovo State Power University, Ivanovo, Russia.

ph.+7(4932)26-97-09, e-mail: klgn@drive.ispu.ru

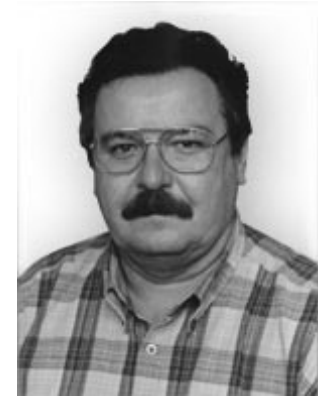

Sergey K. Lebedev in 1975 received Engineer's degree in Ivanovo Power Institute. In 1986 he received Candidate's degree in electrical engineering for the dissertation "Design and Research of Devices of Coordinates and Parameters Definition for the Induction Electric Drive with Vector Control based on Current and Stator Voltage Sensors".

Associate Professor of Electric Drive and Automation of Industrial Plants Department, Ivanovo State Power University, Ivanovo, Russia.

e-mail: lebedev@drive.ispu.ru ph. +7(4932)26-97-09,

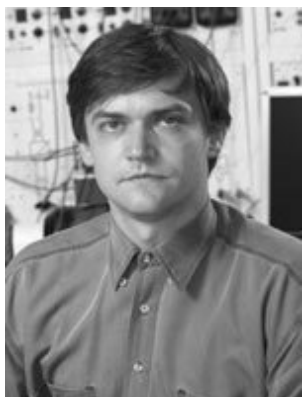

Mikhail S. Kulenko in 1994 received Engineer's degree in Ivanovo Power Institute. In 2002 he received Candidate's degree in electrical engineering for the dissertation "Control system of fiber axial winding on the basis of a roll predictive model".

Associate Professor of Electric Drive and Automation of Industrial Plants Department, Ivanovo State Power University, Ivanovo, Russia.

ph. +7(4932)26-97-09,

e-mail: forestgummy@gmail.com 